Pacific Journal of Mathematics

THE CHARACTER SPACE OF THE ALGEBRA OF REGULATED 


\title{
THE CHARACTER SPACE OF THE ALGEBRA OF REGULATED FUNCTIONS
}

\author{
S. K. BERBERIAN
}

\begin{abstract}
The character space of the algebra of regulated functions on a closed interval is computed and is identified with the character space of the algebra of functions of bounded variation. The dual space of the Banach space of regulated functions is analyzed in terms of the character space.
\end{abstract}

1. Introduction. The regulated complex functions on a compact interval form a commutative $C^{*}$-algebra with unity. The objective of this paper is to explore the character space of this algebra, and those of several related Banach algebras. E. Hewitt's computation of the character space of one of the related algebras [7] is given a new interpretation ( $\$ 4)$. In pre-Banach algebra times, H. S. Kaltenborn gave an elegant description of the dual space of the algebra of regulated functions [10]; we re-examine his results from a Banach algebra point of view, recasting slightly his formula for the continuous linear forms $(\S 6)$.

Let $I=[a, b]$ be a nondegenerate closed interval of the real line $\boldsymbol{R}$, fixed for the rest of the paper. A complex-valued function $f$ on $I$ is said to be regulated if it possesses (finite) one-sided limits at every point of $I$; that is, the limits

$$
f(c+)=\lim _{x \rightarrow c, x>c} f(x), \quad f(c-)=\lim _{x \rightarrow c, x<c} f(x)
$$

exist in $C$ for every $c \in[a, b)$ and every $c \in(a, b]$, respectively. Every regulated function on $I$ is bounded. We write $\mathscr{R}=\mathscr{R}(I)$ for the set of all regulated functions $f: I \rightarrow C$. With the pointwise operations and the norm $\|f\|_{\infty}=\sup _{x \in I}|f(x)|, \mathscr{R}$ is a commutative $C^{*}$-algebra with unity [16, p. 276, Def. 11.17]: completeness for the norm metric follows at once from the "iterated limits theorem" [15, p. 149, Th. 7.11]. To enlarge the perspective a bit, $\mathscr{R}$ is a closed $*$-subalgebra of the commutative $C^{*}$-algebra $\mathscr{B}=\mathscr{B}(I)$ of all bounded complex functions on $I$. In turn, the algebra $\mathscr{C}=\mathscr{C}(I)$ of all continuous complex functions on $I$ is a closed subalgebra of $\mathscr{R}$; thus $\mathscr{C} \subset \mathscr{R} \subset$ $\mathscr{B}$.

A complex function $f$ on $I$ is called a step function if there exists a partition of $I$ into finitely many subintervals (possibly degenerate) on each of which $f$ is constant. We write $\mathscr{S}=\mathscr{S}(I)$ for the algebra of all step functions on $I$, and $\mathscr{B} \mathscr{V}=\mathscr{B} \mathscr{V}(I)$ for the algebra of all complex functions on $I$ of bounded variation. It 
is clear that $\mathscr{S} \subset \mathscr{B} \mathscr{V}$ and that $\mathscr{S}$ is the linear span of the set of characteristic functions $\varphi_{J}$ of subintervals $J$ of $I$; and $\mathscr{B} \mathscr{F} \subset \mathscr{R}$ because, by the Jordan theorem, $\mathscr{B} \mathscr{V}$ is the linear span of the monotone functions (which are evidently regulated). Thus $\mathscr{S} \subset$ $\mathscr{B} \mathscr{V} \subset \mathscr{R}$; since $\mathscr{S}$ is dense in $\mathscr{R}$ for the sup-norm topology [2, Ch. II, $\left.\S 1, n^{\circ} 3\right], \mathscr{R}$ is the completion of both $\mathscr{S}$ and $\mathscr{B} \mathscr{P}$ for this norm. \{In particular, $\mathscr{B} \mathscr{V}$ is not complete for this norm. In $\S 5$ we study $\mathscr{B} \mathscr{V}$ as a Banach algebra relative to another norm.\}

2. Some decompositions of regulated functions. Regulated functions have a certain capacity for self-improvement:

Proposition 1. Let $f \in \mathscr{R}$. Define $f^{*}: I \rightarrow C$ by the formulas

$$
f^{*}(x)=f(x+) \text { for } x \in[a, b), \quad f^{*}(b)=f(b-) .
$$

Then $f^{*} \in \mathscr{R}$. More precisely: (i) $f^{*}$ is right-continuous on $[a, b)$; (ii) $f^{*}$ is left-continuous at $b$; (iii) $f^{*}(x-)=f(x-)$ for all $x \in(a, b]$; (iv) $\left\|f^{*}\right\|_{\infty} \leqq\|f\|_{\infty}$; (v) $f^{*}(x)=f(x)$ for all but denumerably many values of $x$.

Proof. The motivation for the definition of $f^{*}(b)$ is that, when considering possible discontinuities of $f$ at the endpoints $a, b$, it is convenient to extend $f$ to the interval $[a-1, b+1]$ by defining $f(x)=f(a+)$ on $[a-1, a)$ and $f(x)=f(b-)$ on $(b, b+1]$. The extended function will then have at most removable discontinuities at $a, b$, and $f^{*}$ is continuous at these points.

(iv) is obvious, and ( $\mathrm{v}$ ) is immediate from the fact that $f$ is continuous at all but denumerably many points of $I[2, \mathrm{Ch} . \mathrm{II}, \S 1$, Th. 3].

(i) Let $c \in[a, b), c<c_{n}<b, c_{n} \rightarrow c$. For each $n$, choose $x_{n}, c_{n}<$ $x_{n}<b$, such that $\left|f\left(x_{n}\right)-f\left(c_{n}+\right)\right|<1 / n$ and $\left|x_{n}-c_{n}\right|<1 / n$; then $x_{n} \rightarrow c$, therefore $f\left(x_{n}\right) \rightarrow f(c+)$, therefore $\left|f(c+)-f\left(c_{n}+\right)\right| \rightarrow 0$.

(iii) is proved similarly, and implies (ii).

The mapping $P: \mathscr{R} \rightarrow \mathscr{R}$ defined by

$$
P f=f^{*} \quad(f \in \mathscr{R})
$$

is an algebra $*$-homomorphism, continuous for the sup-norm topology, idempotent $\left(P^{2}=P\right)$. We write

$$
\mathscr{R}^{*}=P(\mathscr{R})
$$

for the range of $P$, and 


$$
\mathscr{N}=\operatorname{ker} P
$$

for the kernel of $P$; thus $\mathscr{N}$ is a closed ideal of $\mathscr{R}$, and $\mathscr{R}^{*}=$ $\operatorname{ker}(1-P)$ is a closed $*$-subalgebra of $\mathscr{R}$ that contains $\mathscr{C}$. (Remark: It is easy to see that $\|P\|=1$ and $\|1-P\|=2$. $\}$ Every $f \in \mathscr{R}$ has a unique decomposition $f=g+h$ with $g \in \mathscr{R}^{*}$ (that is, $P g=g$ ) and $h \in \mathscr{N}$ (that is, $P h=0$ ), namely $g=P f, h=f-P f$.

COROLLARY 1. (i) $\mathscr{R}^{*}$ is the set of all $g \in \mathscr{R}$ such that $g$ is right-continuous on $[a, b)$ and left-continuous at $b$; (ii) $\mathscr{N}$ is the set of all $h \in \mathscr{R}$ such that $h(x+)=0$ for all $x \in[a, b)$ and $h(x-)=0$ for all $x \in(a, b]$.

Proof. This is clear from Proposition 1 and the definition of $f^{*}$.

From (ii) of Corollary 1, one sees that for $h \in \mathscr{N}$, the set of discontinuities of $h$ is the set $\{x \in I: h(x) \neq 0\}$.

CoROLlary 2. (i) $\mathscr{N}$ is the closed linear span in $\mathscr{R}$ of the characteristic functions $\varphi_{|x|}, x \in I$; (ii) $\mathscr{R}^{*}$ is the closed linear span in $\mathscr{R}$ of the characteristic functions $\varphi_{J}$, where $J$ is a subinterval of $I$ such that either $J=[c, d)$ with $d<b$, or $J=[c, b]$.

Proof. (i) If $f=\varphi_{J}, J$ a subinterval of $I$, then $f^{*}$ can differ from $f$ only at the endpoints of $J$; therefore if $f$ is a step function, then $(1-P) f=f-f^{*}$ vanishes at all but finitely many points (hence is a linear combination of functions $\varphi_{\{x\}}$ ). Suppose $h \in \mathscr{N}$. Choose a sequence of step functions $f_{n}$ such that $f_{n} \rightarrow h$ uniformly in $I$; then $(1-P) f_{n} \rightarrow(1-P) h=h$ uniformly, whence (i).

(ii) Let $\mathscr{J}$ be the indicated set of subintervals of $I$; these are the subintervals $J$ of $I$ such that $\varphi_{J} \in \mathscr{R}^{*}$. Let $\mathscr{R}_{0}$ be the closed linear span of the $\varphi_{J}, J \in \mathscr{J}$; evidently $\mathscr{R}_{0} \subset \mathscr{R}^{*}$. If $f$ is the characteristic function of a subinterval of $I$ with endpoints $c, d$, then $f^{*}$ is the characteristic function of the interval in $\mathscr{J}$ with endpoints $c, d$; therefore $f^{*} \in \mathscr{R}_{0}$ whenever $f$ is a step function. Suppose $g \in$ $\mathscr{R}^{*}$. Choose a sequence of step functions $f_{n}$ such that $f_{n} \rightarrow g$ uniformly in $I$; then $f_{n}^{*} \rightarrow g^{*}=g$ uniformly, whence $g \in \mathscr{R}_{0}$.

E. Hewitt studied the algebra of restrictions to $[a, b)$ of the functions in $\mathscr{R}^{*}$; identifying the two algebras in the obvious way, part (ii) of Corollary 2 corresponds to Theorem 4.5 of his paper [7, p. 87]. Here are some useful characterizations of $\mathscr{N}$ :

CoROLlaRY 3. The following conditions on a function $f: I \rightarrow C$ are equivalent: 
(a) $f \in \mathscr{N}$;

(b) $f \in \mathscr{R}, f(x+)=0$ for all $x \in[a, b)$;

(b') $f \in \mathscr{R}, f(x-)=0$ for all $x \in(a, b]$;

(c) $f \in \mathscr{R}$ and the set $\{x \in I: f(x) \neq 0\}$ is denumerable;

(d) $f \in \mathscr{R}$ and the set $\{x \in I: f(x)=0\}$ is dense in $I$;

(e) there exist a sequence $c_{n} \in I$ and a sequence $r_{n} \in C$ with $r_{n} \rightarrow 0$, such that $f\left(c_{n}\right)=r_{n}$ for all $n$ and $f(x)=0$ for all other $x$.

Proof. $\quad(\mathrm{a}) \Longrightarrow(\mathrm{b}),\left(\mathrm{b}^{\prime}\right)$ by Corollary 1 .

$(\mathrm{b}) \Longrightarrow(\mathrm{c}),\left(\mathrm{b}^{\prime}\right) \Longrightarrow(\mathrm{c})$ : Clear from the fact that every $f \in \mathscr{R}$ is continuous at all but denumerably many points of $I$.

$(c) \Longrightarrow(\mathrm{d})$ : Obvious.

$(d) \Longrightarrow(e)$ : From (d), it is clear that (b), (b') hold, hence also (c). To prove (e), it will suffice to show that, given any $\varepsilon>0$, the set $\{x \in I:|f(x)| \geqq \varepsilon\}$ is finite. Assume to the contrary that $\left|f\left(x_{n}\right)\right| \geqq \varepsilon$ for a (faithfully indexed) infinite sequence $x_{n}$. Passing to a subsequence, we can suppose that $x_{n}$ is convergent, say $x_{n} \rightarrow x$, and that either $x_{n}<x$ for all $n$ or $x_{n}>x$ for all $n$; in either case, $f\left(x_{n}\right) \rightarrow 0$ by (b) or $\left(\mathrm{b}^{\prime}\right)$, contrary to $\left|f\left(x_{n}\right)\right| \geqq \varepsilon$.

$(\mathrm{e}) \Rightarrow(\mathrm{a})$ : In the notations of (e), let $f_{n}=\sum_{k=1}^{n} \boldsymbol{r}_{k} \varphi_{\left\{c_{k}\right\}}$; then $\left\|f-f_{n}\right\|_{\infty} \rightarrow 0$, therefore $f \in \mathscr{N}$ by (i) of Corollary 2.

Dually, one can define, for each $f \in \mathscr{R}$, a function $* f \in \mathscr{R}$ by the formulas

$$
* f(x)=f(x-) \text { for } x \in(\alpha, b], \quad * f(\alpha)=f(\alpha+) .
$$

Defining Q: $\mathscr{R} \rightarrow \mathscr{R}$ by

$$
Q f=* f \quad(f \in \mathscr{R}),
$$

one sees that $Q$ has properties analogous to those of $P$; in view of the symmetry in Corollary 3 of Proposition 1 , the kernel of $Q$ is also $\mathscr{N}$; we write ${ }^{*} \mathscr{R}=Q(\mathscr{R})$, which is the set of all $f \in \mathscr{R}$ that are left-continuous on $(a, b]$ and right-continuous at $a$. In view of Proposition 1 and its dual, inspection of the definitions yields the formulas

$$
P Q=P, \quad Q P=Q
$$

WWe remark that if $f \in \mathscr{R}$ is real-valued, then the upper and lower semicontinuous regularizations of $f$ [1, Ch. IV, $\left.\S 6, n^{\circ} 2\right]$, namely the functions $\bar{f}=\lim \sup f, \underline{f}=\lim \inf f$, are given by the formulas $\bar{f}=\max \{f, P f, Q f\}, \underline{f}=\min \{f, P f, Q f\}$, hence are obviously regulated. Writing $g=\bar{f}, h=\bar{f}-\bar{f}$, one obtains a decomposition $f=g+h$ with $g \in \mathscr{R}$ upper semicontinuous and $h \in \mathscr{N}$ (by criterion (c) of Corollary 3 
of Proposition 1). However, such decompositions are not unique, nor do they possess the algebraic properties enjoyed by the decompositions described earlier; they will play no role in the rest of the paper.\}

3. The character space of $\mathscr{R}$. A character of a commutative Banach algebra $\mathscr{A}$ is an algebra epimorphism $\mathscr{A} \rightarrow \boldsymbol{C}$. The set $X(\mathscr{A})$ of all characters of $\mathscr{A}$, equipped with the topology of simple convergence in $\mathscr{A}$, is a locally compact space, called the character space of $\mathscr{A}$ (this topology on $X(\mathscr{A})$ is called the Gelfand topology); when $\mathscr{A}$ has a unity element, $X(\mathscr{A})$ is compact [4, Ch. I, §3]. Since $\mathscr{R}$ is a commutative $C^{*}$-algebra with unity, it is isomorphic to the algebra $\mathscr{C}(X(\mathscr{R}))$ of all continuous complex functions on the compact space $X(\mathscr{R})[4, \mathrm{Ch} . \mathrm{I}, \S 6$, Th. 1]; our central objective is to explore the space $X(\mathscr{R})$.

\section{THEOREM 1. $X(\mathscr{R})$ is totally disconnected.}

Proof. Let $\mathscr{J}$ be the family of all subintervals $J$ of $I$. The mapping $\beta \mapsto\left(\beta\left(\varphi_{J}\right)\right)_{J \in}$ is a continuous mapping of $X(\mathscr{R})$ into a cartesian product $\mathscr{X}$ of copies of the discrete space $\{0,1\}$; it is injective because $\mathscr{S}$ is dense in $\mathscr{R}$; since $X(\mathscr{R})$ is compact and $\mathscr{Q}$ is separated, the mapping is a homeomorphism of $X(\mathscr{R})$ onto a subspace of the totally disconnected space $\mathscr{X}$.

THEOREM 2. $X(\mathscr{R})$ does not have a denumerable base for open sets (hence is not metrizable).

Proof. Since $\mathscr{C}(X(\mathscr{R}))$ is isomorphic to $\mathscr{R}$, it suffices to prove that the Banach algebra $\mathscr{R}$ is not separable [1, Ch. X, $\S 3$, Th. 1]. This is shown by the family of functions $f_{x}=\varphi_{\{x\}}, x \in I$, which satisfies $\left\|f_{x}-f_{y}\right\|_{\infty}=1$ for $x \neq y$.

The closed open sets of $X(\mathscr{R})$ (there are lots of them, by Theorem 1) correspond, via the Gelfand isomorphism, to the idempotents in the algebra $\mathscr{R}$. The idempotents of $\mathscr{R}$ are all in $\mathscr{S}$ :

THEOREM 3. The idempotents of $\mathscr{R}$ are the characteristic functions $\varphi_{A}$, where $A$ is the union of finitely many subintervals of $I$.

Proof. Let $f \in \mathscr{R}$ be idempotent, say $f=\varphi_{A}, A \subset I$. Let $c \in$ $[a, b)$ and consider open intervals $J \subset I$ with left endpoint $c$; if one had both $J \cap A \neq \varnothing$ and $J \cap(I-A) \neq \varnothing$ for every such $J$, then $f(c+)$ would fail to exist, a contradiction. Thus, for every $c \in[a, b)$, there exists an open interval $J_{c} \subset I$ with left endpoint $c$, such that either $J_{c} \subset A$ or $J_{c} \subset I-A$. Similarly, for each $c \in(a, b]$, there exists 
an open interval $K_{c} \subset I$ with right endpoint $c$, such that either $K_{c} \subset A$ or $K_{c} \subset I-A$. Defining

$$
U_{a}=\{a\} \cup J_{a}, U_{b}=K_{b} \cup\{b\}, U_{c}=K_{c} \cup\{c\} \cup J_{c} \text { for } c \in(a, b),
$$

one has an open covering of the compact space $I$; say $I=U_{x_{1}} U$ $U_{x_{2}} \cup \cdots \cup U_{x_{n}}$; then $A=\left(A \cap U_{x_{1}}\right) \cup \cdots \cup\left(A \cap U_{x_{n}}\right)$, and each $A \cap U_{x_{i}}$ is either an interval or the union of two intervals.

CoRollary. In the lattice of closed open subsets of $X(\mathscr{R})$, there exists a denumerable family that does not have a supremum.

Proof. Let $c_{n}$ be a sequence in $I$ such that $c_{1}<c_{2}<c_{3}<\cdots$ and $c_{n} \rightarrow b$. Let $J_{n}=\left[c_{n}, c_{n+1}\right)$ and $f_{n}=\varphi_{J_{n}}$. Assuming that the corollary is false, let $f$ (resp. $g$ ) be the supremum of the $f_{n}$ for $n$ odd (resp. even). \{Caution: We mean here supremum in the lattice sense, which must be distinguished from the pointwise supremum.\} Say $f=\varphi_{A}, g=\varphi_{B}$. For $m \neq n$ one has $f_{m} f_{n}=0$, thus $f_{m} \leqq 1-f_{n}$; it follows that $f g=0$, therefore $A \cap B=\varnothing$. By Theorem $3, A$ is the disjoint union of a finite number of intervals; since $A \supset J_{n}$ for all odd $n$, the rightmost nondegenerate interval in this representation of $A$ has right endpoint $b$ (which it need not contain). Similarly, $B$ contains a nondegenerate interval with right endpoint $b$. Then $A \cap B$ contains a nondegenerate interval, contrary to $A \cap B=\varnothing$.

We write $\beta, \gamma, \delta$ for generic elements of $X(\mathscr{R})$; there are the obvious characters

$$
\begin{array}{ll}
\beta_{x}(f)=f(x) & \text { for } f \in \mathscr{R}, \\
\delta_{x}(f)=f(x+) & \text { for } f \in \mathscr{R}, \\
\gamma_{x}(f)=f(x-) & \text { for } f \in \mathscr{R},
\end{array}
$$

defined for $x \in I, x \in[a, b)$ and $x \in(a, b]$, respectively; we shall see in Theorem 5 that there are no others.

TheOREM 4. Define $\Psi: I \rightarrow X(\mathscr{R})$ by $\Psi(x)=\delta_{x}$ for $x \in[a, b)$ and $\Psi(b)=\gamma_{b}$. Then $\Psi$ is right-continuous on $[a, b)$ and left-continuous at $b$.

Proof. Let $f \in \mathscr{R}$. For all $x \in I$ one has $\Psi(x)(f)=f^{*}(x)$, thus the theorem is immediate from $\S 2$, Proposition 1.

THEOREM 5.

$$
X(\mathscr{R})=\left\{\beta_{x}: x \in[a, b]\right\} \cup\left\{\delta_{x}: x \in[a, b)\right\} \cup\left\{\gamma_{x}: x \in(a, b]\right\} .
$$

Proof. Let $\beta \in X(\mathscr{R})$ and assume to the contrary that $\beta$ does 
not have one of the three indicated forms, equivalently, that $\operatorname{ker} \beta$ is not equal to (hence is not contained in) $\operatorname{ker} \beta_{x}, \operatorname{ker} \delta_{x}$ or $\operatorname{ker} \gamma_{x}$, for any $x$. For each $x \in I$, choose $f_{x} \in \operatorname{ker} \beta$ with $f_{x}(x) \neq 0$; replacing $f_{x}$ by $\bar{f}_{x} f_{x}$, we can suppose that $f_{x} \geqq 0, f_{x}(x)>0$. For each $x \in[a, b)$, choose $g_{x} \in \operatorname{ker} \beta$ with $g_{x} \geqq 0$ and $g_{x}(x+)>0$; let $J_{x} \subset I$ be an open interval with left endpoint $x$, such that $g_{x}$ is bounded away from zero on $J_{x}$. Similarly, for each $x \in(a, b]$, there exist $h_{x} \in \operatorname{ker} \beta, h_{x} \geqq 0$, and an open interval $K_{x} \subset I$ with right endpoint $x$, such that $h_{x}$ is bounded away from zero on $K_{x}$. For $x \in(a, b)$ define

$$
V_{x}=K_{x} \cup\{x\} \cup J_{x}, \quad k_{x}=h_{x}+f_{x}+g_{x},
$$

and define $V_{a}=\{a\} \cup J_{a}, k_{a}=f_{a}+g_{a}, V_{b}=K_{b} \cup\{b\}, k_{b}=h_{b}+f_{b}$. For every $x \in I, V_{x}$ is a neighborhood of $x$ in $I$, and $k_{x}$ is a positive function, belonging to $\operatorname{ker} \beta$, such that $k_{x}$ is bounded away from zero on $V_{x}$. Say $I=V_{x_{1}} \cup \cdots \cup V_{x_{n}}$. Then the function $k=k_{x_{1}}+\cdots+k_{x_{n}}$ belongs to $\operatorname{ker} \beta$ and is bounded away from zero on $I$; it follows that $1 / k$ is regulated, whence $1=(1 / k) k \in \operatorname{ker} \beta$, which is absurd.

\section{Corollary 1. $X(\mathscr{R})$ has the cardinality of the continuum.}

We write $\alpha$ for generic elements of $X(\mathscr{C})$, specifically $\alpha_{x}(f)=$ $f(x)$ for $x \in I, f \in \mathscr{C}$; the mapping $I \rightarrow X(\mathscr{C})$ defined by $x \mapsto \alpha_{x}$ is a homeomorphism [4, Ch. I, $\S 3$, Cor. 2 of Prop. 1]. If $\beta \in X(\mathscr{R})$, then $\beta \mid \mathscr{C} \in X(\mathscr{C})$, thus $\beta \mid \mathscr{C}=\alpha_{x}$ for a unique $x \in I$; defining $\Phi(\beta)=$ $x$, we have a continuous surjection

$$
\Phi: X(\mathscr{R}) \longrightarrow I, \quad \alpha_{\Phi(\beta)}=\beta \mid \mathscr{C} \text { for } \beta \in X(\mathscr{R}) .
$$

For the mapping $\Psi$ of Theorem 4 , one has $\Phi \circ \Psi=\mathrm{id}_{I}$; so to speak, $\Psi$ is a right-continuous cross-section for $\Phi$. \{No continuous crosssection exists, since $X(\mathscr{R})$ contains no connected subset with more than one element.\}

CoROLLARY 2. $\Phi^{-1}(\{a\})=\left\{\beta_{a}, \delta_{a}\right\}, \Phi^{-1}(\{b\})=\left\{\beta_{b}, \gamma_{b}\right\}$, and $\Phi^{-1}(\{x\})=$ $\left\{\beta_{x}, \gamma_{x}, \delta_{x}\right\}$ for $x \in(a, b)$.

COROLLARY 3. If $\xi \in X(\mathscr{B})$, there exists $c \in I$ satisfying one of the following three conditions: (i) $\xi(f)=f(c)$ for all $f \in \mathscr{R}$; $\xi(f)=f(c+)$ for all $f \in \mathscr{R}$; (iii) $\xi(f)=f(c-)$ for all $f \in \mathscr{R}$.

Proof. Let $\beta=\xi \mid \mathscr{R}$ and cite Theorem 5 .

Here is another perspective on Corollary 3. Regard $I_{d}$ ( $I$ with the discrete topology) as a dense subspace of its Stone-Čech com- 
pactification $X(\mathscr{B})$. Every bounded function $f: I \rightarrow C$ may be uniquely extended to a continuous function $\hat{f}: X(\mathscr{B}) \rightarrow C$. The message of Corollary 3: for each $\xi \in X(\mathscr{B})$, there exists $c \in I$ such that one of the following three conditions holds: (i) $\hat{f}(\xi)=f(c)$ for all $f \in \mathscr{R}$; (ii) $\hat{f}(\xi)=f(c+)$ for all $f \in \mathscr{R}$; (iii) $\hat{f}(\xi)=f(c-)$ for all $f \in \mathscr{R}$. \{We remark that all three cases occur, since the mapping $X(\mathscr{B}) \rightarrow X(\mathscr{R})$ defined by restriction of characters is surjective $[13$, p. 126, (3.2.16)].\}

CoRollaRY 4. For every $x \in I,\left\{\beta_{x}\right\}$ is an isolated point of $X(\mathscr{R})$.

Proof. It is clear from Theorem 5 that

$$
\left\{\beta_{x}\right\}=\left\{\beta \in X(\mathscr{R}):\left|\beta\left(\varphi_{\{x\}}\right)-\beta_{x}\left(\varphi_{\{x\}}\right)\right|<1\right\} .
$$

Theorem 2 is an obvious consequence of Corollary 4. The proof of the next corollary employs the 'hull-kernel' characterization of the topology of $X(\mathscr{R})$. If $\mathscr{A}$ is any commutative Banach algebra, the Gelfand topology on $X(\mathscr{A})$ is finer than the hull-kernel topology; when the two topologies coincide, $\mathscr{A}$ is said to be completely regular. Every commutative $C^{*}$-algebra is completely regular [13, p. 174, (3.7.2)]. In particular, $\mathscr{R}$ is completely regular; thus, if $S$ is any subset of $X(\mathscr{R})$, the closure of $S$ is given by the formula

$$
\bar{S}=h k(S)=\left\{\beta^{\prime} \in X(\mathscr{R}): \operatorname{ker} \beta^{\prime} \supset \bigcap_{\beta \in S} \operatorname{ker} \beta\right\} .
$$

Corollary 5. Let $X(\mathscr{R})=E \cup F \cup G$ be the partition of $X(\mathscr{R})$ defined by

$$
E=\left\{\beta_{x}: x \in[a, b]\right\}, \quad F=\left\{\delta_{x}: x \in[a, b)\right\}, G=\left\{\gamma_{x}: x \in(a, b]\right\} .
$$

Then $E$ is a dense, open, discrete subspace of $X(\mathscr{R})$, and

$$
\bar{F}=\bar{G}=F \cup G=X(\mathscr{R})-E
$$

is a perfect subset of $X(\mathscr{R})$ with empty interior.

Proof. That $E$ is dense results from $\bigcap_{x \in I} \operatorname{ker} \beta_{x}=\{0\}$, thus it is clear from Corollary 4 that $E$ has the properties claimed for it. From $\S 2$, Corollary 3 of Proposition 1 , one sees that $k(F)=\mathscr{N}=k(G)$, therefore $\bar{F}=h k(F)=h k(G)=\bar{G}$; since $F \cup G=C E$ is closed, it follows that $F \cup G=\overline{F \cup G}=\bar{F} \cup \bar{G}=\bar{F}=\bar{G}$. Evidently $F \subset \bar{G}$ and $G \subset \bar{F}$, therefore $F \cup G$ is dense in itself. Since $F \cup G$ is disjoint from the dense set $E$, its interior is empty.

From Corollaries 4 and 5 , one sees that a subset of $X(\mathscr{R})$ is 
dense if and only if it contains $E$, and that $X(\mathscr{R})$ has $2^{c}$ open subsets. The second assertion of Corollary 5 is an instance of a theorem of A. Pelczyinski and Z. Semadeni: a compact space $T$ contains a nonempty perfect subset if and only if there exists a continuous surjection $T \rightarrow I$ ([12], [11, p. 29, Th. 2]). Incidentally, $X(\mathscr{R})$ is not the StoneČech compactification of its dense open subspace $E$; for, the Stone-Čech compactification of a discrete space is extremally disconnected [19, p. 300], and $X(\mathscr{R})$ is not extremally disconnected (by the corollary of Theorem 3 [cf. 17, p. 185, Th. 14]). \{Alternative proof: $\mathscr{R}$ has $c$ idempotents (Theorem 3 ), whereas $\mathscr{B}$ has $2^{c}$ idempotents, thus $\mathscr{R}$ and $\mathscr{B}$ are not isomorphic.\} The fact that $X(\mathscr{R})$ contains a dense subspace $E$ homeomorphic to $I_{d}$ ( $I$ with the discrete topology) was predictable from [5, p. 225, Th. 1], since $\mathscr{R}$ contains the functions $\varphi_{\langle x\rangle}$.

An explicit formula for the closure operation in $X(\mathscr{R})$ is given in Corollary 7; the following notations prepare the way. For a subset $A$ of $I$, we write $\mathscr{J}_{A}$ for the kernel of the set $\left\{\beta_{x}: x \in A\right\}$, that is,

$$
\mathscr{F}_{A}=\bigcap_{x \in A} \operatorname{ker} \beta_{x}=\{f \in \mathscr{R}: f \mid A=0\} .
$$

We write $A_{+}^{\prime}$ for the set of $x \in I$ for which there exists a sequence $x_{n} \in A$ such that $x_{n}>x$ and $x_{n} \rightarrow x$, thus

$$
A_{+}^{\prime}=\{x \in I: x \in \overline{(x,+\infty) \cap A}\} ;
$$

this is, so to speak, the 'right derived set' of $A$. Similarly, we write

$$
A_{-}^{\prime}=\{x \in I: x \in \overline{(-\infty, x) \cap A}\} \text {. }
$$

Then $A_{+}^{\prime} \cup A_{-}^{\prime}=A^{\prime}$, the usual derived set of $A$. One has $(A \cup B)_{+}^{\prime}=$ $A_{+}^{\prime} \cup B_{+}^{\prime},(A \cup B)_{-}^{\prime}=A_{-}^{\prime} \cup B_{-}^{\prime}$. If $J$ is a subinterval of $I$ with endpoints $c, d(c \leqq d)$, then $J_{+}^{\prime}=[c, d)$ and $J_{-}^{\prime}=(c, d]$.

Lemma 1. Let $A \subset I, S=\left\{\beta_{x}: x \in A\right\}, c \in I$. Then: (i) $\beta_{c} \in \bar{S}$ if and only if $c \in A$; (ii) $\delta_{c} \in \bar{S}$ if and only if $c \in A_{+}^{\prime}$; (iii) $\gamma_{c} \in \bar{S}$ if and only if $c \in A_{-}^{\prime}$.

Proof. (ii) Suppose $\delta_{c} \in \bar{S}$ (in particular, $c<b$ ), that is, ker $\delta_{c} \supset$ $k(S)=\mathscr{J}_{A}$. Assuming to the contrary that $c \notin A_{+}^{\prime}$, choose an open interval $J \subset I$ with left endpoint $c$, such that $A \cap J=\varnothing$; then $\varphi_{J} \mid A=0$ but $\varphi_{J}(c+)=1$, contrary to the supposition that $\mathscr{F}_{A} \subset \operatorname{ker} \delta_{c}$. Conversely, suppose $c \in A_{+}^{\prime}$ and choose a sequence $c_{n} \in A$ such that $c_{n}>c$ and $c_{n} \rightarrow c$. For every $f \in \mathscr{R}, f\left(c_{n}\right) \rightarrow f(c+)$; if, moreover, $f \in \mathscr{J}_{A}$, then $f\left(c_{n}\right)=0$ for all $n$, whence $f(c+)=0, f \in \operatorname{ker} \delta_{c}$.

(iii) is proved similarly, and (i) is obvious. 
LeMma 2. Let $A \subset I, S=\left\{\beta_{x}: x \in A\right\}$. Then $\bar{S}=S \cup\left\{\delta_{x}: x \in A_{+}^{\prime}\right\} \cup$ $\left\{\gamma_{x}: x \in A_{-}^{\prime}\right\}$.

Proof. Immediate from Lemma 1 and Theorem 5.

COROLlaRY 6. The closed open subsets of $X(\mathscr{R})$ are the sets of the form $\bar{S}_{A}$, where $A$ is the union of finitely many subintervals of $I$ and $S_{A}=\left\{\beta_{x}: x \in A\right\}$.

Proof. The closed open sets in $X(\mathscr{R})$ correspond to the idempotents of $\mathscr{R}$ via the Gelfand isomorphism. By Theorem 3, the idempotents of $\mathscr{R}$ are the functions $\varphi_{A}, A$ the union of finitely many subintervals of $I$. For such an $A$, the closed open subset of $X(\mathscr{R})$ corresponding to $\varphi_{A}$ is the set $U(A)=\left\{\beta \in X(\mathscr{R}): \beta\left(\varphi_{A}\right)=1\right\}$; evidently $\beta_{x}\left(\varphi_{A}\right)=1$ if and only if $x \in A ; \delta_{x}\left(\varphi_{A}\right)=1$ if and only if $x \in A_{+}^{\prime}$; and $\gamma_{x}\left(\varphi_{A}\right)=1$ if and only if $x \in A_{-}^{\prime}$; therefore

$$
U(A)=\left\{\beta_{x}: x \in A\right\} \cup\left\{\delta_{x}: x \in A_{+}^{\prime}\right\} \cup\left\{\gamma_{x}: x \in A_{-}^{\prime}\right\},
$$

thus $U(A)=\bar{S}_{A}$ by Lemma 2 .

From Corollary 6 (or Theorem 3) one sees that $X(\mathscr{R})$ has $c$ closed open sets; a closed open subset of $X(\mathscr{R})$ either is a finite subset of $E$ or has cardinality $c$.

Lemma 3. Let $B \subset[a, b), S=\left\{\delta_{x}: x \in B\right\} . \quad$ Then $\bar{S} \cap F=S \cup$ $\left\{\delta_{x}: x \in B_{+}^{\prime}\right\}$.

Proof. Let $x \in[a, b)$; the problem is to show that $\delta_{x} \in \bar{S} \Leftrightarrow x \in$ $B \cup B_{+}^{\prime}$.

Proof of $\Rightarrow$ : Suppose $x \notin B \cup B_{+}^{\prime}$. Since $x<b$ and $x \notin B_{+}^{\prime}$ there exists $y, x<y<b$, such that $B \cap(x, y)=\varnothing$. Since $x \notin B$, also $B \cap[x, y)=\varnothing$. Let $J=[x, y), f=\varphi_{J}$. If $t \in B$ then $t<x$ or $t \geqq y$, and in either case $f(t+)=0$; thus $f \in \bigcap_{t \in B} \operatorname{ker} \delta_{t}=k(S)$. But $f(x+)=1$, thus $\operatorname{ker} \delta_{x} \not \supset k(S)$, that is, $\delta_{x} \notin \bar{S}$.

Proof of $\Longleftarrow:$ Suppose $x \in B_{+}^{\prime}$. Choose a sequence $x_{n} \in B$ such that $x_{n}>x$ and $x_{n} \rightarrow x$. Then $\delta_{x_{n}} \rightarrow \delta_{x}$ by Theorem 4, thus $\delta_{x} \in \bar{S}$.

Lemma 4. Let $B \subset[a, b), S=\left\{\delta_{x}: x \in B\right\}$. Then $\bar{S} \cap G=\left\{\gamma_{x}: x \in B_{-}^{\prime}\right\}$.

Proof. Let $x \in(a, b]$; the problem is to show that $\gamma_{x} \in \bar{S} \Leftrightarrow x \in B_{-}^{\prime}$.

Proof of $\Rightarrow$ : Suppose $x \notin B_{-}^{\prime}$. Then, since $a<x$, there exists 
$y, a<y<x$, such that the interval $J=[y, x)$ is disjoint from $B$. Let $f=\varphi_{J}$. If $t \in B$ then $t<y$ or $t \geqq x$, and in either case $f(t+)=0$; thus $f \in k(S)$. But $f(x-)=1$, thus $\operatorname{ker} \gamma_{x} \not \supset k(S)$, that is, $\gamma_{x} \notin \bar{S}$.

Proof of $\Longleftarrow:$ Suppose $x \in B_{-}^{\prime}$. Choose a sequence $x_{n} \in B$ such that $x_{n}<x, x_{n} \rightarrow x$. If $f \in k(S)$ then $f\left(x_{n}\right)=0$ for all $n$; thus, in the notation of $\S 2$, Proposition 1 , one has $f^{*}\left(x_{n}\right)=0$ and $\gamma_{x}(f)=f(x-)=$ $f^{*}(x-)=\lim f^{*}\left(x_{n}\right)=0$. Thus $\operatorname{ker} \gamma_{x} \supset k(S)$, that is, $\gamma_{x} \in \bar{S}$.

Lemma 5. Let $B \subset[a, b), S=\left\{\delta_{x}: x \in B\right\}$. Then

$$
\bar{S}=S \cup\left\{\delta_{x}: x \in B_{+}^{\prime}\right\} \cup\left\{\gamma_{x}: x \in B_{-}^{\prime}\right\} .
$$

Proof. Since $S \subset F$, one has $\bar{S} \subset \bar{F}=F \cup G$, therefore $\bar{S}=(\bar{S} \cap F) \cup$ $(\bar{S} \cap G)$; cite Lemmas 3 and 4 .

By dual arguments one shows:

Lemma 6. Let $C \subset(a, b], S=\left\{\gamma_{x}: x \in C\right\}$. Then

$$
\bar{S}=S \cup\left\{\gamma_{x}: x \in C_{-}^{\prime}\right\} \cup\left\{\delta_{x}: x \in C_{+}^{\prime}\right\} \text {. }
$$

CoRollary 7. Given any set $S \subset X(\mathscr{R})$, say

$$
S=\left\{\beta_{x}: x \in A\right\} \cup\left\{\delta_{x}: x \in B\right\} \cup\left\{\gamma_{x}: x \in C\right\},
$$

where $A \subset[a, b], B \subset[a, b), C \subset(a, b]$. Then

$$
\bar{S}=S \cup\left\{\delta_{x}: x \in(A \cup B \cup C)_{+}^{\prime}\right\} \cup\left\{\gamma_{x}: x \in(A \cup B \cup C)_{-}^{\prime}\right\} \text {. }
$$

In particular, $S$ is closed if and only if $(A \cup B \cup C)_{+}^{\prime} \subset B$ and $(A \cup B \cup C)_{-}^{\prime} \subset C$.

Proof. This is immediate from Lemmas 2, 5, and 6, and the 'additivity' of the derived set operations.

Here is an amusing consequence of Corollaries 6 and 7 . Let $A \subset I$. In order that $A$ be the union of finitely many intervals, it is necessary and sufficient that there exist partitions $I=A_{1} \cup A_{2}$, $[a, b)=B_{1} \cup B_{2},(a, b]=C_{1} \cup C_{2}$, with $A=A_{1}$, such that $\left(A_{i} \cup B_{i} \cup C_{i}\right)_{+}^{\prime} \subset B_{i}$ and $\left(A_{i} \cup B_{i} \cup C_{i}\right)_{-}^{\prime} \subset C_{i}$ for $i=1,2$.

The next proposition is for application in $\S 6$. Let $\Phi: X(\mathscr{R}) \rightarrow I$ be the mapping (11); let $\beta \sim \beta^{\prime}$ denote the equivalence relation defined in $X(\mathscr{R})$ by $\Phi(\beta)=\Phi\left(\beta^{\prime}\right)$ (that is, by $\beta\left|\mathscr{C}=\beta^{\prime}\right| \mathscr{C}$ ), write $\Lambda: X(\mathscr{R}) \rightarrow$ $X(\mathscr{R}) / \sim$ for the quotient mapping, and equip $X(\mathscr{R}) / \sim$ with the quotient topology. The equivalence classes for $\sim$ are the sets $\Phi^{-1}(\{x\})$, 
$x \in I$ (cf. Cor. 2 of Th. 5). Let $\Phi^{\prime}: X(\mathscr{R}) / \sim \rightarrow I$ be the continuous bijection derived from $\Phi$ by passage to quotients (thus $\Phi=\Phi^{\prime} \circ \Lambda$ ); it follows from compactness that $\Phi^{\prime}$ is a homeomorphism [1, Ch. I, §9, Cor. 2 of Th. 2]. Finally, if $\Gamma: \mathscr{R} \rightarrow \mathscr{C}(X(\mathscr{R}))$ is the Gelfand isomorphism, then for every $f \in \mathscr{C}=\mathscr{C}(I)$, one has $\Gamma f=f \circ \Phi$. \{Proof: For all $\beta \in X(\mathscr{R}),(\Gamma f)(\beta)=\beta(f)=(\beta \mid \mathscr{C})(f)=\alpha_{\Phi(\beta)}(f)=f(\Phi(\beta))=$ $(f \circ \Phi)(\beta)$.

Proposition 2. For a function $u: X(\mathscr{R}) \rightarrow C$, the following conditions are equivalent:

(a) $u=f \circ \Phi$ for some continuous $f: I \rightarrow C$;

(b) $u=\Gamma g$ for some continuous $g: I \rightarrow C$;

(c) $u$ is continuous, and is constant on each of the sets $\Phi^{-1}(\{x\})$, $x \in I$.

In the notations of (a) and (b), necessarily $f=g$.

Proof. The remark immediately preceding the proposition proves the equivalence of (a) and (b), as well as the final assertion of the proposition. It is obvious that (a) implies (c).

$(c) \Rightarrow(a)$ : Since $u$ is constant on each set $\Phi^{-1}(\{x\})$, there exists a factorization $u=f \circ \Phi$ with $f: I \rightarrow C$; from the continuity of $u$, we are to infer the continuity of $f$. Since $\left(f \circ \Phi^{\prime}\right) \circ \Lambda=f \circ\left(\Phi^{\prime} \circ \Lambda\right)=f \circ \Phi=u$ is continuous, and since $X(\mathscr{R}) / \sim$ bears the quotient topology, it follows that $f \circ \Phi^{\prime}$ is continuous; but $\Phi^{\prime}$ is a homeomorphism, therefore $f$ is continuous.

4. The character spaces of $\mathscr{R}^{*}$ and $\mathscr{N}$. The notations $P, Q$, $\mathscr{N}, \mathscr{R}^{*},{ }^{*} \mathscr{R}$. have the same meanings as in $\S 2$, and $X(\mathscr{R})=E \cup F \cup G$ is the partition described in $\S 3$, Corollary 5 of Theorem 5 . The commutative $C^{*}$-algebras $\mathscr{R}, \mathscr{N}, \mathscr{R}^{*}$ are related by the isomorphism $\mathscr{R}^{*} \cong \mathscr{R} / \mathscr{N}$; the relation between their character spaces is given by the following general lemma [cf. 13, p. 193, Th. 4.2.4]:

Lemma. If $T$ is a compact space, $S$ is a closed subset of $T$, and $\mathscr{F}$ is the ideal of $\mathscr{C}(T)$ annihilating $S$, then $\mathscr{C}(T) / \mathscr{J} \cong \mathscr{C}(S)$ and $\mathscr{I} \cong \mathscr{C}_{0}(T-S)$, therefore $X(\mathscr{C}(T) / \mathscr{I})=S$ and $X(\mathscr{J})=T-S$.

Proof. Consider the mappings $\mathscr{C}(T) \rightarrow \mathscr{C}(S)$ and $\mathscr{F} \rightarrow \mathscr{C}_{0}(T-S)$ defined by $f \mapsto f \mid S$ and $f \mapsto f \mid T-S$ and cite, respectively, the Tietze extension theorem and the Stone-Weierstrass theorem. (Here $\mathscr{C}_{0}(T-S)$ denotes the algebra of continuous functions vanishing at infinity on the locally compact space $T-S$.\}

The first assertion of the following theorem was proved by E. Hewitt [7, p. 91, Th. 5.1]: 
THEOREM 6. $X\left(\mathscr{R}^{*}\right)$ is homeomorphic to $F \cup G ; X(\mathscr{N})$ is homeomorphic to $E$, that is, to $I_{d}$ (I equipped with the discrete topology).

Proof. Identifying $\mathscr{R}$ (via the Gelfand isomorphism) with $\mathscr{C}(X(\mathscr{R})), \mathscr{N}$ is the ideal of $\mathscr{R}$ annihilating the closed set $F \cup G$ ( $\{2$, Cor. 1 of Prop. 1). Cite the lemma. (It is easy to see that the homeomorphisms $E \rightarrow X(\mathscr{N}), F \cup G \rightarrow X\left(\mathscr{R}^{*}\right)$ are effected by restriction of characters. Note, incidentally, that $\delta_{x}\left|\mathscr{R}^{*}=\beta_{x}\right| \mathscr{R}^{*}$ for $x \in[a, b)$, and $\left.\gamma_{b}\left|\mathscr{R}^{*}=\beta_{b}\right| \mathscr{R}^{*}.\right\}$

The same reasoning shows that $X\left(^{*} \mathscr{R}\right)$ is homeomorphic to $F \cup G$. This reminds us that $* \mathscr{R}$ and $\mathscr{R}^{*}$ are isomorphic; indeed, it follows from the relations $\mathscr{R}^{*}=P(\mathscr{R})=P\left({ }^{*} \mathscr{R}+\mathscr{N}\right)=P\left({ }^{*} \mathscr{R}\right)$, that $P$ effects an isomorphism of ${ }^{*} \mathscr{R}$ onto $\mathscr{R}^{*}$. In view of Theorem 6 , the message of $\S 2$, Corollary 3 of Proposition 1 is that $\mathscr{N}=\mathscr{C}_{0}\left(I_{d}\right)$.

5. The character space of $\mathscr{B} \mathscr{Y}$. It will be shown that $\mathscr{B} \mathscr{V}$ has the same character space as $\mathscr{R}$; first, we see that $\mathscr{B} \mathscr{V}$ can be normed to be a Banach algebra $[13$, p. 302]:

LEMma. Equipped with pointwise operations and the norm

$$
\|f\|=V_{a}^{b} f+\|f\|_{\infty},
$$

$\mathscr{B} \mathscr{Y}$ is a commutative, involutive Banach algebra with unity.

Proof. Here $V_{a}^{b} f$ denotes the total variation of $f$ in $I[8$, p. 266, (17.14)]. Let $f, g \in \mathscr{B} \mathscr{P}$; from the identity

$$
(f g)(x)-(f g)(y)=f(x)[g(x)-g(y)]+[f(x)-f(y)] g(y)
$$

one sees that $V_{a}^{b}(f g) \leqq\|f\|_{\infty} V_{a}^{b} g+\|g\|_{\infty} V_{a}^{b} f$ (this shows, in particular, that $f g \in \mathscr{B} \mathscr{Y})$; it follows that the norm (18) satisfies $\|f g\| \leqq\|f\|\|g\|$. Completeness for this norm is easily deduced from [9, p. 43, Th. 8.6].

THEOREM 7. The mapping $X(\mathscr{R}) \rightarrow X(\mathscr{B} \mathscr{V})$ defined by $\beta \mapsto$ $\beta \mid \mathscr{B} \mathscr{Y}$ is a homeomorphism.

Proof. The mapping is continuous (for the Gelfand topologies) and injective (e.g., because $\mathscr{B} \mathscr{V}$ is dense in $\mathscr{R}$ for the sup-norm topology). It will suffice (by compactness) to show that it is surjective. Let $\varepsilon \in X(\mathscr{B} \mathscr{V})$ and suppose to the contrary that $\varepsilon \neq \beta \mid \mathscr{B} \mathscr{Y}$, equivalently $\operatorname{ker} \varepsilon \not \subset \operatorname{ker} \beta$, for all $\beta \in X(\mathscr{R})$. Arguing exactly as in the proof of Theorem 5, one constructs $k \in$ ker $\varepsilon$ such that $k$ is bounded away from zero in $I$. It is then immediate from the identity 


$$
(1 / k)(x)-(1 / k)(y)=[k(y)-k(x)] / k(x) k(y)
$$

that $1 / k \in \mathscr{B} \mathscr{V}$; whence $1=(1 / k) k \in$ ker $\varepsilon$, which is absurd.

It follows that the closure operation in $X(\mathscr{B} \mathscr{Y})$ is also given by the formula in Corollary 7 of Theorem 5 .

\section{THEOREM 8. $\mathscr{B} \mathscr{V}$ is completely regular.}

Proof. As is true for every commutative Banach algebra with unity, $X(\mathscr{B} \mathscr{Y})$ is compact for the Gelfand topology and quasicompact for the hull-kernel topology [4, Ch. I, §1, Prop. 4], and the Gelfand topology is finer than the hull-kernel topology; to prove that the two topologies coincide, it will suffice to show that the hull-kernel topology is separated. To this end, it suffices $[6, \mathrm{p} .111,7 \mathrm{M}]$ to show that if $\varepsilon_{1}, \varepsilon_{2}$ are distinct characters of $\mathscr{B} \mathscr{V}$, then there exist functions $f_{1}, f_{2}$ in $\mathscr{B} \mathscr{V}$ such that $\varepsilon_{1}\left(f_{1}\right)=\varepsilon_{2}\left(f_{2}\right)=1$ and $f_{1} f_{2}=0$. In view of Theorem 7, one is reduced to the consideration of a small number of cases. For example, suppose $\varepsilon_{1}=\beta_{x} \mid \mathscr{B} \mathscr{C}$ and $\varepsilon_{2}=\delta_{y} \mid \mathscr{B} \mathscr{V}$; if $x \leqq y$, take $f_{1}=\varphi_{\{x\}}$ and $f_{2}=\varphi_{\{y, b)} ;$ if $x>y$, take $f_{1}=\varphi_{\{x\}}$ and $f_{2}=\varphi_{(y, x)}$. The remaining cases are equally transparent. \{We remark that $\mathscr{B} \mathscr{V}$ is not isomorphic (as an algebra) to a $C^{*}$-algebra; for, the spectral radius of $f \in \mathscr{B} \mathscr{Y}$ is $\|f\|_{\infty}$, and $\mathscr{B} \mathscr{C}$ is not complete for this norm.\}

An example due to G. Šilov [cf. 13, p. 302, A.2.5] fits into the present circle of ideas. Let us write $\mathscr{B} \mathscr{V}_{C}$ for the subalgebra $\mathscr{C} \cap$ $\mathscr{B} \mathscr{V}$ of $\mathscr{B} \mathscr{V}$. It is elementary that $\mathscr{B} \mathscr{V} c$ is closed in $\mathscr{B} \mathscr{V}$ for the norm (18), hence is a Banach algebra for this norm.

THEOREM 9 (̌̌silov). $X\left(\mathscr{B} \mathscr{V}_{c}\right)$ is homeomorphic to $X(\mathscr{C})$ (that is, to $I)$.

Proof. Recall that $X(\mathscr{C})=\left\{\alpha_{x}: x \in I\right\}$, where $\alpha_{x}(f)=f(x)$ for $f \in \mathscr{C}$. The mapping $X(\mathscr{C}) \rightarrow X\left(\mathscr{B} \mathscr{V}_{c}\right)$ defined by $\alpha \mapsto \alpha \mid \mathscr{B}_{\mathscr{C}}$ is continuous and injective; to prove that it is a homeomorphism, it suffices to show that it is surjective. Let $\varepsilon \in X\left(\mathscr{B} \mathscr{C}_{c}\right)$ and assume to the contrary that $\varepsilon \neq \alpha_{x} \mid \mathscr{B}_{c}$ for all $x \in I$, that is, $\operatorname{ker} \varepsilon \not \subset \operatorname{ker} \alpha_{x}$ for all $x \in I$. Repeating a classical argument (simpler than that in Theorem 5), one constructs a function $k \in \operatorname{ker} \varepsilon$ such that $k$ vanishes at no point of $I$; since $k$ is continuous, it is bounded away from zero; therefore $1 / k \in \mathscr{B} \mathscr{\mathscr { V }}$, whence $1=(1 / k) k \in \operatorname{ker} \varepsilon$, which is absurd. \{Incidentally, by a simplification of the argument in Theorem 8 , one sees that $\mathscr{B} \mathscr{V}_{c}$ is completely regular $[13$, p. 302]. (There is only one case to consider: $\varepsilon_{1}=\alpha_{x}\left|\mathscr{B} \mathscr{V}_{c}, \varepsilon_{2}=\alpha_{y}\right| \mathscr{B}_{c} \mathscr{V}_{c}$ with $\left.\left.x \neq y \cdot\right)\right\}$ 
Next, we study the decomposition $f=P f+(1-P) f$ of $\S 2$ for functions of bounded variation.

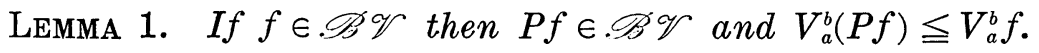

Proof. Let $f \in \mathscr{B} \mathscr{Y}$ and let $a=x_{0}<x_{1}<\cdots<x_{n}=b$ be any subdivision of $I$; we are to show that $\sum_{i=1}^{n}\left|f^{*}\left(x_{i}\right)-f^{*}\left(x_{i-1}\right)\right| \leqq V_{a}^{b} f$. Choose points $y_{i}, 1 \leqq i \leqq n+1$, such that $x_{i-1}<y_{i}<x_{i}$ for $1 \leqq i \leqq$ $n-1$ and $x_{n-1}<y_{n}<y_{n+1}<x_{n}=b$. Then $V_{a}^{b} f \geqq \sum_{i=2}^{n+1}\left|f\left(y_{i}\right)-f\left(y_{i-1}\right)\right|$; the desired inequality results on letting $y_{i} \rightarrow x_{i-1}$ for $1 \leqq i \leqq n$ and $y_{n+1} \rightarrow b$.

It follows from Lemma 1 that $P(\mathscr{B} \mathscr{Y})=\mathscr{R}^{*} \cap \mathscr{B} \mathscr{V}$, and that $\|P f\| \leqq\|f\|$ for all $f \in \mathscr{B} \mathscr{C}$; the restriction of $P$ to $\mathscr{B} \mathscr{V}$ is an algebra $*$-homomorphism $P_{0}: \mathscr{B} \mathscr{V} \rightarrow \mathscr{B} \mathscr{V}$, continuous for the norm \| $\|$, idempotent; therefore, in the Banach algebra $(\mathscr{B} \mathscr{Y}\|\|), \operatorname{ker} P_{0}=$ $\mathscr{N} \cap \mathscr{B} \mathscr{V}$ is a closed ideal and $P_{0}(\mathscr{B} \mathscr{V})=\mathscr{R}^{*} \cap \mathscr{B} \mathscr{V}$ is a closed *-subalgebra. Passing to quotients, one has an isomorphism

$$
\mathscr{B} \mathscr{V} /(\mathscr{N} \cap \mathscr{B} \mathscr{Y}) \cong \mathscr{R}^{*} \cap \mathscr{B} \mathscr{V} \text {; }
$$

it follows from Theorem 8 that the algebras $\mathscr{N} \cap \mathscr{B} \mathscr{V}$ and $\mathscr{R}^{*} \cap$ $\mathscr{B} \mathscr{O}$ are also completely regular $[13$, p. 84, (2.7.2)]. [We remark that the upper and lower semicontinuous regularizations of a realvalued function in $\mathscr{B} \mathscr{V}$ are also in $\mathscr{B} \mathscr{C}$; this is immediate from the formulas mentioned at the end of §2.\} Here are some useful characterizations of $\mathscr{N} \cap \mathscr{B} \mathscr{V}$ :

LEMma 2. The following conditions on a function $f: I \rightarrow C$ are equivalent:

(a) $f \in l^{1}(I)$, that is, $\|f\|_{1}=\sum_{x \in I}|f(x)|<+\infty$;

(b) $f \in \mathscr{N} \cap \mathscr{B} \mathscr{V}$;

(c) $f \in \mathscr{B} \mathscr{V}$ and the set $\{x \in I: f(x) \neq 0\}$ is denumerable;

(d) $f \in \mathscr{B} \mathscr{Y}$ and the set $\{x \in I: f(x)=0\}$ is dense in $I$.

For such a function $f$ one has

$$
\|f\|_{1} \leqq V_{a}^{b} f \leqq 2\|f\|_{1}
$$

Proof. $\quad(a) \Rightarrow(b)$ : If $f \in l^{1}(I)$, it is obvious that $V_{a}^{b} f \leqq 2\|f\|_{1}<$ $+\infty$, therefore $f \in \mathscr{B} \mathscr{V} \subset \mathscr{R}$, and the set $\{x \in I: f(x) \neq 0\}$ is denumerable, thus $f \in \mathscr{N}$ by $\S 2$, Corollary 3 of Proposition 1 .

(b) $\Rightarrow$ (c) is immediate from $\S 2$, Corollary 3 of Proposition 1 , and $(c) \Rightarrow(d)$ is obvious.

$(\mathrm{d}) \Rightarrow(\mathrm{a})$ : Let $f \in \mathscr{B} \mathscr{C}$ and suppose that the set $D=\{x \in I: f(x)=0\}$ is dense in $I$. Consider any finite subset of $I$, say $x_{1}<x_{2}<\cdots<x_{n}$. 
Choose $y_{1}, \cdots, y_{n} \in D$ such that $y_{i} \in\left(x_{i}, x_{i+1}\right)$ for $1 \leqq i \leqq n-2$ and $x_{n-1}<y_{n-1}<y_{n}<x_{n}$; then

$$
V_{a}^{b} f \geqq \sum_{i=1}^{n-1}\left|f\left(x_{i}\right)-f\left(y_{i}\right)\right|+\left|f\left(y_{n}\right)-f\left(x_{n}\right)\right|=\sum_{i=1}^{n}\left|f\left(x_{i}\right)\right|,
$$

thus $f \in l^{1}(I)$ and $\|f\|_{1} \leqq V_{a}^{b} f$.

LEMma 3. $\mathscr{N} \cap \mathscr{B} \mathscr{Y}$ is the closed linear span in $\mathscr{B} \mathscr{P}$ of the functions $\varphi_{\{x\}}, x \in I$, for the norm (18).

Proof. Let $\mathscr{F}$ be the linear span of the functions $\varphi_{\{x\}}, x \in I$. Since $\mathscr{N} \cap \mathscr{B} \mathscr{O}$ is closed for the norm \|\| (remarks following Lemma 1), it contains the closure of $\mathscr{F}$. Conversely, suppose $f \in$ $\mathscr{N} \cap \mathscr{B} \mathscr{Y}$. By Lemma 2, there exists a sequence $f_{n} \in \mathscr{F}$ such that $\left\|f_{n}-f\right\|_{1} \rightarrow 0$, and $V_{a}^{b}\left(f_{n}-f\right) \rightarrow 0$ by (19); since $\left\|f_{n}-f\right\|_{\infty} \leqq\left\|f_{n}-f\right\|_{1}$, it follows that $\left\|f_{n}-f\right\| \rightarrow 0$.

THEOREM 10. (i) $X(\mathscr{N} \cap \mathscr{B} \mathscr{Y})$ is homeomorphic to $E$, that is, to $I_{d}$ (I with the discrete topology); (ii) $X\left(\mathscr{R}^{*} \cap \mathscr{B} \mathscr{V}\right.$ ) is homeomorphic to $F \cup G$.

Proof. (i) \{To put it less obliquely, $X\left(\mathscr{N}^{*} \cap \mathscr{B} \mathscr{P}\right)$ is discrete and consists of the point evaluations.\} Suppose $\varepsilon \in X(\mathscr{N} \cap \mathscr{B} \mathscr{V})$; by Lemma 3 , there exists $x \in I$ such that $\varepsilon\left(\varphi_{\{x \mid}\right)=1$; for $y \neq x$, $1+\varepsilon\left(\varphi_{\{y\}}\right)=\varepsilon\left(\varphi_{\{x\}}+\varphi_{\{y\}}\right)=\varepsilon\left(\varphi_{\{x, y\}}\right)$ is 0 or 1 , therefore $\varepsilon\left(\varphi_{\{y\}}\right)=0$; it follows from Lemma 3 that $\varepsilon=\beta_{x} \mid \mathscr{N} \cap \mathscr{B} \mathscr{V}$. Thus, the mapping $E \rightarrow X(\mathscr{N} \cap \mathscr{B} \mathscr{\mathscr { C }})$ defined by $\beta \mapsto \beta \mid \mathscr{N} \cap \mathscr{B} \mathscr{P}$ is bijective, and $X(\mathscr{N} \cap \mathscr{B} \mathscr{V})$ is discrete by the proof of $\S 3$, Corollary 4 of Theorem 5 .

(ii) If $\varepsilon \in X\left(\mathscr{R}^{*} \cap \mathscr{B} \mathscr{V}\right)$ then $\varepsilon \circ P_{0} \in X(\mathscr{B} \mathscr{V})$, therefore (Theorem 7) there exists $\beta \in F \cup G$ such that $\beta \mid \mathscr{B} \mathscr{P}=\varepsilon \circ P_{0}$, whence $\beta \mid \mathscr{R}^{*} \cap$ $\mathscr{B} \mathscr{V}=\varepsilon$. Thus, the continuous mapping $F \cup G \rightarrow X\left(\mathscr{R}^{*} \cap \mathscr{B} \mathscr{V}\right)$ defined by restriction of characters is surjective; to show that it is a homeomorphism, it suffices to show that it is injective. Let $\beta_{1}, \beta_{2} \in$ $F \cup G, \beta_{1} \neq \beta_{2}$; we seek $f \in \mathscr{R}^{*} \cap \mathscr{B} \mathscr{Y}$ such that $\beta_{1}(f) \neq \beta_{2}(f)$. If $\beta_{1}, \beta_{2}$ are induced by the same point $x$ of $I$, say $\beta_{1}=\gamma_{x}$ and $\beta_{2}=\delta_{x}$, then $a<x<b$ and we may take $f=\varphi_{[a, x)}$. Suppose $\beta_{1}, \beta_{2}$ are induced by distinct points $x, y$ of $I, x<y$; if $\beta_{1}=\gamma_{x}$ (and $\beta_{2}=\gamma_{y}$ or $\delta_{y}$ ), take $f=\varphi_{[a, x]}$; if $\beta_{1}=\delta_{x}$ (and $\beta_{2}=\gamma_{y}$ or $\hat{\delta}_{y}$ ), take $f=\varphi_{[x, r]}$ for any $r$ such that $x<r<y$.

6. The dual spaces of $\mathscr{R}, \mathscr{R}^{*}, \mathscr{N}$. The dual space $\mathscr{R}^{\prime}$ of $\mathscr{R}$ has been calculated by H. S. Kaltenborn [10]; we review here his formula for the continuous linear forms on $\mathscr{R}$. To conform to his notations, we write $T$ for elements of $\mathscr{R}^{\prime}, \chi$ for elements of $\mathscr{B} \mathscr{Y}$, 
and $\phi$ for elements of $l^{1}(I)$ (by $\S 5$, Lemma 2, these are the functions in $\mathscr{N} \cap \mathscr{\mathscr { B }} \mathscr{Y})$. Each $\chi \in \mathscr{B} \mathscr{V}$ defines $T_{\chi} \in \mathscr{R}^{\prime}$ by the formula

$$
T_{\chi} f=\int_{a}^{b} f d \chi \text { for } f \in \mathscr{R},
$$

where the right side is the 'modified Stieltjes integral' whose existence was proved by B. Dushnik (see [10]): it is the limit, in the sense of refinement of subdivisions $a=x_{0}<x_{1}<\cdots<x_{n}=b$ of $I$, of the sums

$$
\sum_{i=1}^{n} f\left(\xi_{i}\right)\left[\chi\left(x_{i}\right)-\chi\left(x_{i-1}\right)\right],
$$

where $\xi_{i}$ is required to be a point of the open interval $\left(x_{i-1}, x_{i}\right)$. On the other hand, if $\phi \in l^{1}(I)$ then the formula

$$
T_{\phi} f=\sum_{x \in I}[f(x)-f(x+)] \phi(x) \quad \text { for } \quad f \in \mathscr{R}
$$

defines $T_{\phi} \in \mathscr{R}^{\prime}$. Note that $f(b+)=f(b-)$ by the convention pertaining to the definition of $f$ on $(b, b+1]$ : see the remarks in the proof of $\S 2$, Proposition 1.\} Caution: The subscript on $T$ determines the formula of definition. The following lemma is readily verified:

Lemma. (i) If $\chi \in \mathscr{B} \mathscr{P}$ then $T_{\chi} \mid \mathscr{N}=0$ and $\left\|T_{\chi}\right\|=V_{a}^{b} \chi=$ $\left\|T_{\chi} \mid \mathscr{R}^{*}\right\| . \quad$ (ii) If $\phi \in l^{1}(I)$ then $T_{\phi} \mid \mathscr{R}^{*}=0,\left\|T_{\phi}\right\|=2\|\phi\|_{1}$ and $\left\|T_{\phi} \mid \mathscr{N}\right\|=\|\phi\|_{1}$

THEOREM 11 (Kaltenborn). Every $T \in \mathscr{R}^{\prime}$ has a representation $T=T_{\chi}+T_{\phi}$, where $\chi \in \mathscr{B} \mathscr{Y}$ and $\phi \in l^{1}(I) ; \phi$ is unique and $\chi$ is unique up to an additive constant.

If, in Theorem 11, one requires that $\chi \in \mathscr{B} \mathscr{V}_{0}$ (the functions in $\mathscr{B} \mathscr{\mathscr { V }}$ that vanish at $\alpha$ ), then $\chi$ is uniquely determined by $T$. Note that $V_{a}^{b} \chi$ is a norm on $\mathscr{B} \mathscr{V}_{0}$.

\section{CoRollaRY 1. $\left(\mathscr{R}^{*}\right)^{\prime}=\mathscr{B} \mathscr{V}_{0}$.}

Proof. If $\chi \in \mathscr{B} \mathscr{V}_{0}$ then $T_{\chi} \mid \mathscr{R}^{*} \in\left(\mathscr{R}^{*}\right)^{\prime}$ and $\left\|T_{\chi} \mid \mathscr{R}^{*}\right\|=V_{a}^{b} \chi$ by the lemma. Conversely, if $\lambda \in\left(\mathscr{R}^{*}\right)^{\prime}$ then $T=\lambda \circ P \in \mathscr{R}^{\prime}$ and $T \mid \mathscr{N}=$ 0 ; therefore by Kaltenborn's theorem there exists a unique $\chi \in \mathscr{B} \mathscr{V}_{0}$ such that $T=T_{\chi}$, whence $T_{\chi} \mid \mathscr{R}^{*}=\lambda$. $\quad\left\{\right.$ In particular, $\mathscr{B} \mathscr{V}_{0}$ is a Banach space for the norm $\left.V_{a}^{b} \chi[8, \mathrm{p} .271,(17.35)].\right\}$

E. Hewitt has shown that $\left(\mathscr{R}^{*}\right)^{\prime}$ may be represented as the space of all bounded, finitely additive measures defined on the ring of subsets of $I$ generated by the intervals of the form $[c, d), a \leqq c<d \leqq b$ $[7$, p. 90 , Th. 4.10]. 
Corollary 2. $\mathscr{N}^{\prime}=l^{1}(I)$.

Proof. If $\phi \in l^{1}(I)$ then $T_{\phi} \mid \mathscr{N} \in \mathscr{N}^{\prime}$ and $\left\|T_{\phi} \mid \mathscr{N}\right\|=\|\phi\|_{1}$. Conversely, if $\mu \in \mathscr{N}^{\prime}$ then $T=\mu \circ(1-P) \in \mathscr{R}^{\prime}$ and $T \mid \mathscr{R}^{*}=0$; therefore by Kaltenborn's theorem there exists a unique $\phi \in l^{1}(I)$ such that $T=T_{\phi}$, whence $T_{\phi} \mid \mathscr{N}=\mu$. \{Alternate proof: Since $\mathscr{N}=\mathscr{C}_{0}\left(I_{d}\right)$ ( $\$$, Cor. 3 of Prop. 1 ), it is elementary that $\mathscr{N}^{\prime}=l^{1}(I)$. $\}$

Thus, the structure of $\mathscr{R}^{\prime}$ is as follows: given $\lambda \in\left(\mathscr{R}^{*}\right)^{\prime}$ and $\mu \in \mathscr{N}^{\prime}$, one forms $T=\lambda \circ P+\mu \circ(1-P)$; this is the general element of $\mathscr{R}^{\prime}$. One can also deduce Theorem 5 from Kaltenborn's formula; the computations are tedious but thoroughly elementary (nothing so fancy as the Gelfand theory is needed).

Since $\mathscr{R}$ is isomorphic to $\mathscr{C}(X(\mathscr{R})), \mathscr{R}^{\prime}$ may be identified with the space $\mathscr{C}(X(\mathscr{R}))$ of measures on $X(\mathscr{R})$. The rest of the paper is devoted to the measure-theoretic description of $\mathscr{R}^{\prime}$.

By measure we mean complex (Radon) measure [3, Ch. III, $\S 1$, Def. 2] (equivalently, complex regular Borel measure). The measures on $I$ are the Lebesgue-Stieltjes measures induced by functions of bounded variation ([8, p. 331, (19.48)], [14, p. 263]). The mapping $\Phi: X(\mathscr{R}) \rightarrow I$ described in formula (11) will figure in the following results. If $\mu$ is a measure on $X(\mathscr{R})$, the image of $\mu$ under $\Phi$ is the measure $\nu=\Phi(\mu)$ on $I$ defined by $\nu(f)=\mu(f \circ \Phi)$ for $f \in \mathscr{C}$. For every measure $\nu$ on $I$, there exist measures $\mu$ on $X(\mathscr{R})$ such that $\Phi(\mu)=\nu$, and if $\nu$ is positive then $\mu$ can be chosen to be positive [3, Ch. IX, p. 33, Lemma 1]. Thus, the mapping $\mathscr{C}(X(\mathscr{R})) \rightarrow \mathscr{C}(I)$ defined by $\mu \mapsto \Phi(\mu)$ is surjective; it is linear, positive and contractive; in view of $\S 3$, Proposition 2, its kernel is the subspace $\Gamma(\mathscr{C})^{\perp}$, consisting of all measures $\mu$ on $X(\mathscr{R})$ that are zero on the image of $\mathscr{C}=\mathscr{C}(I)$ under the Gelfand isomorphism $\Gamma: \mathscr{R} \rightarrow \mathscr{C}(X(\mathscr{R}))$. We remark that the quotient mapping $\mathscr{C}(X(\mathscr{R})) / \Gamma(\mathscr{C})^{\perp} \rightarrow \mathscr{M}(I)$ is isometric. (Proof: Identify $\mathscr{R}$ with $\mathscr{C}(X(\mathscr{R}))$ via $\Gamma$; the mapping $\mu \mapsto \Phi(\mu)$ then becomes the mapping $\mathscr{R}^{\prime} \rightarrow \mathscr{C}^{\prime}$ defined by $\mu \mapsto \mu \mid \mathscr{C}$, with kernel $\mathscr{C}^{1}$. The quotient mapping $\mathscr{R}^{\prime} / \mathscr{C}^{\perp} \rightarrow \mathscr{C}^{\prime}$ is an isometric vector space isomorphism [16, p. 91 , Th. 4.9].\}

THEOREM 12. (i) $X(\mathscr{R})$ admits nonzero diffuse measures. (ii) The support of every diffuse measure on $X(\mathscr{R})$ is contained in $F \cup G$. (iii) $A$ positive measure $\mu$ on $X(\mathscr{R})$ is diffuse if and only if $\Phi(\mu)$ is diffuse.

Proof. A measure on a space is said to be diffuse if every onepoint subset of the space is negligible. The support of a measure is the complement of the largest negligible open set in the space. 
(iii) Let $\mu$ be a positive measure on $X(\mathscr{R})$ and let $\nu=\Phi(\mu)$. For every $x \in I$, one has $\mu\left(\Phi^{-1}(\{x\})\right)=\nu(\{x\})$ [3, Ch. V, $\S 6$, Cor. 1 of Proof. 2]. Since the $\Phi^{-1}(\{x\})$ form a covering of $X(\mathscr{R})$, it is clear that the diffuseness of $\nu$ implies that of $\mu$; since the sets $\Phi^{-1}(\{x\})$ are finite ( $\$ 3$, Cor. 2 of Th. 5), the converse is also true. (The diffuse measures on $I$ are the Lebesgue-Stieltjes measures induced by continuous functions of bounded variation $[8$, p. 332, (19.52)].\}

(i) Let $\nu$ be a nonzero, diffuse positive measure on $I$ (for instance, Lebesgue measure), let $\mu$ be a positive measure on $X(\mathscr{R})$ such that $\Phi(\mu)=\nu$, and cite (iii). \{In view of $\S 3$, Corollary 5 of Theorem 5, this is a manifestation of a general theorem of A. Pelczynski and $Z$. Semadeni: a compact space admits a nonzero diffuse measure if and only if it has a nonempty perfect subset ([12, p. 214], [11, p. 52, Th. 10]).\}

(ii) Let $\mu$ be a diffuse measure on $X(\mathscr{R})$. Replacing $\mu$ by $|\mu|$, we can suppose that $\mu$ is positive. Since the set $E$ in Corollary 5 of Theorem 5 is open, one has $\mu(E)=\sup \{\mu(K): K \subset E, K$ compact $\}$ [3, Ch. IV, $\S 4$, Cor. 4 of Th. 4]; every compact subset $K$ of the discrete space $E$ is finite, hence is negligible by the hypothesis on $\mu$; therefore $\mu(E)=0$, thus $E \subset \complement$ Supp $\mu$.

To each $T \in \mathscr{R}^{\prime}$ there corresponds a unique measure $\mu_{T}$ on $X(\mathscr{R})$ such that $T f=\mu_{T}(\Gamma f)$ for all $f \in \mathscr{R}(\Gamma$ the Gelfand isomorphism); $T \mapsto \mu_{T}$ is an isometric, positivity-preserving vector space isomorphism $\mathscr{R}^{\prime} \rightarrow \mathscr{H}(X(\mathscr{R}))=\mathscr{C}(X(\mathscr{R}))^{\prime}$. If, in particular, $T=T_{\chi}$ for $\chi \epsilon$ $\mathscr{B} \mathscr{V}$ (resp. $T=T_{\phi}$ for $\phi \in l^{1}(I)$ ), we write $\mu_{x}$ (resp. $\mu_{\phi}$ ) for $\mu_{T}$.

Lemma 1. $\Phi\left(\mu_{T}\right)=T \mid \mathscr{C}$ for all $T \in \mathscr{R}^{\prime}$.

Proof. Let $T \in \mathscr{R}^{\prime}, \nu=\Phi\left(\mu_{T}\right)$. For all $f \in \mathscr{C}(I)$ one has $\nu(f)=$ $\mu_{T}(f \circ \Phi)=\mu_{T}(\Gamma f)=T f$.

LEMMA 2. (i) For every $\phi \in l^{1}(I), \Phi\left(\mu_{\phi}\right)=0$; (ii) for every $\chi \in$ $\mathscr{B} \mathscr{Y}, \Phi\left(\mu_{x}\right)$ is the Stieltjes integral ('unmodified') induced by $\chi$.

Proof. (i) $T_{\phi} \mid \mathscr{C}=0$ (lemma to Theorem 11).

(ii) For $f \in \mathscr{C}, T_{\chi} f=\int_{a}^{b} f d \chi$ is the limit, in the sense of refinement of subdivisions, of the Riemann-Stieltjes sums $\sum_{i} f\left(\xi_{i}\right)\left[\chi\left(x_{i}\right)-\right.$ $\chi\left(x_{i-1}\right)$, where, since $f$ is continuous, $\xi_{i}$ can be any point of the closed interval $\left[x_{i-1}, x_{i}\right]$.

LEMMA 3. For $T \in \mathscr{R}^{\prime}$, the following conditions are equivalent: (a) Supp $\mu_{T} \subset F \cup G$; (b) $T=T_{\chi}$ with $\chi \in \mathscr{B} \mathscr{Y}$.

Proof. $(a) \Rightarrow(b)$ : By hypothesis, the open set $E$ is contained 
in $\complement$ Supp $\mu_{T}$, therefore $\mu_{T} \mid E=0$. Thus, if $g \in \mathscr{C}(X(\mathscr{R}))$ with Supp $g \subset E$ (in other words, if $g$ is a finite linear combination of characteristic functions $\left.\varphi_{\left(\beta_{x}\right)}\right)$, then $\mu_{T}(g)=0$. In particular, for all $x \in I$ one has $0=\mu_{T}\left(\varphi_{\left\{\beta_{x}\right\}}\right)=\mu_{T}\left(\Gamma \varphi_{\{x\}}\right)=T \varphi_{\{x\}}$, therefore $T=0$ on $\mathscr{N}(\S 2$, Cor. 2 of Prop. 1). Thus, writing $T=T_{\chi}+T_{\phi}$ as in Theorem 11, one has $0=T\left|\mathscr{N}=T_{\chi}\right| \mathscr{N}+T_{\phi}\left|\mathscr{N}=T_{\phi}\right| \mathscr{N}$, whence $\phi=0, T=T_{\chi}$.

(b) $\Rightarrow(a):$ If $T=T_{\chi}, \chi \in \mathscr{B} \mathscr{Y}$, then for all $x \in I$ one has $0=$ $T \phi_{|x|}=\mu_{T}\left(\phi_{\left\{\beta_{x} \mid\right.}\right)$; reversing the preceding argument, we conclude that $\mu_{T} \mid E=0$, thus Supp $\mu_{T} \subset F \cup G$.

Lemma 4. For $T \in \mathscr{R}^{\prime}$, the following conditions are equivalent: (a) $\mu_{T}$ is diffuse; (b) $T=T_{\chi}$ with $\chi \in \mathscr{B}_{\bar{c}}$.

Proof. $\quad(\mathrm{a}) \Rightarrow(\mathrm{b})$ : Write $\mu=\mu_{T}$ and suppose $\mu$ is diffuse. Let $\mu=\mu_{1}-\mu_{2}+i \mu_{3}-i \mu_{4}$ be the canonical decomposition of $\mu$, where the $\mu_{i}$ are positive measures [3, Ch. III, $\S 1$, Th. 3]. The $\mu_{i}$ are also diffuse, therefore Supp $\mu_{i} \subset F \cup G$ by Theorem 12. Say $\mu_{i}=\mu_{T_{i}}$, $T_{i} \in \mathscr{R}^{\prime}$. By Lemma 3, one has $T_{i}=T_{\chi_{i}}$ with $\chi_{i} \in \mathscr{B} \mathscr{Y}$, thus $\mu_{i}=$ $\mu_{x_{i}}$. We can suppose $\chi_{i}(a)=0$; then, since $\mu_{i} \geqq 0$, equivalently $T_{i} \geqq 0$, it follows that $\chi_{i}$ is an increasing real-valued function $\geqq 0$. By Lemma 1, $T_{\chi_{i}}\left|\mathscr{C}=T_{i}\right| \mathscr{C}=\Phi\left(\mu_{T_{i}}\right)=\Phi\left(\mu_{i}\right)$, which is diffuse (Theorem $12)$, therefore $\chi_{i}$ is continuous. Finally, $T=T_{\chi}$, where $\chi=\chi_{1}-\chi_{2}+$ $i \chi_{3}-i \chi_{4}$ is continuous and of bounded variation.

(b) $\Rightarrow(a)$ : Suppose $T=T_{\chi}$ with $\chi \in \mathscr{B} \mathscr{V}_{c}$; one can suppose, by linearity, that $\chi$ is real-valued, increasing and continuous. Then $\Phi\left(\mu_{x}\right)=T_{\chi} \mid \mathscr{C}$ is a diffuse measure on $I[8, \mathrm{p} .332,(19.52)]$, therefore $\mu_{x}$ is diffuse (Theorem 12).

If $\phi \in l^{1}(I)$, a suggestive notation for $T_{\phi}$ is $\sum_{x \in I} \phi(x)\left(\beta_{x}-\delta_{x}\right)$ (with the convention that $\delta_{b}=\gamma_{b}$ ); since $\left\|\beta_{x}-\delta_{x}\right\| \leqq 2$, the sum is convergent for the norm of $\mathscr{R}^{\prime}$. In the same way, one can form 'weighted sums' of arbitrary families in $X(\mathscr{R})$, indexed by $I$, with 'weight function' $\phi \in l^{1}(I)$.

THEOREM 13. Every $T \in \mathscr{R}^{\prime}$ has a representation

$$
T=T_{\psi}+\sum_{x \in[a, b]} \phi_{1}(x) \beta_{x}+\sum_{x \in[a, b)} \phi_{2}(x) \delta_{x}+\sum_{x \in\{a, b]} \phi_{3}(x) \gamma_{x},
$$

where $\psi \in \mathscr{B} \mathscr{C}_{c}$ and $\phi_{1}, \phi_{2}, \phi_{3} \in l^{1}(I)$.

Proof. In view of Theorem 11, it suffices to consider the case that $T=T_{\chi}$ with $\chi \in \mathscr{B} \mathscr{Y}$, and by linearity one can suppose that $\chi$ is real-valued and increasing. Decomposing $\chi$ as the sum of an increasing continuous function $\psi$ and an increasing 'saltus' function 
$[18$, p. 92, 2.4.2], we are reduced to the case that $\chi$ is a saltus function (positive and increasing). Then $\chi=\sum_{n=1}^{\infty} \chi_{n}$, a uniformly convergent series, where $\chi_{n}$ is an increasing step function with a single discontinuity $c_{n}$, say $\chi_{n}=u_{n}^{\prime} \varphi_{\left\{c_{n}\right\}}+\left(u_{n}^{\prime}+u_{n}^{\prime \prime}\right) \varphi_{\left\{c_{n}, b\right]}$, and where $\sum u_{n}^{\prime}, \sum u_{n}^{\prime \prime}$ are convergent positive term series. Since, for each $n$, $\chi-\sum_{k=1}^{n} \chi_{k}=\sum_{k>n} \chi_{k}$ is an increasing function $\psi_{n}$, it follows that $V_{a}^{b}\left(\chi-\sum_{k=1}^{n} \chi_{k}\right)=\psi_{n}(b)-\psi_{n}(a) \rightarrow 0-0$, therefore $T_{\chi} f=\sum_{n=1}^{\infty} T_{\chi_{n}} f$ for all $f \in \mathscr{R}$. Since $T_{x_{n}} f=u_{n}^{\prime} f\left(c_{n}-\right)+u_{n}^{\prime \prime} f\left(c_{n}+\right)$ for all $f \in \mathscr{R}$, one has $T_{\chi_{n}}=u_{n}^{\prime} \gamma_{c_{n}}+u_{n}^{\prime \prime} \delta_{c_{n}}$, therefore $T_{\chi}=\sum u_{n}^{\prime} \gamma_{c_{n}}+\sum u_{n}^{\prime \prime} \delta_{c_{n}}$.

With notation as in Theorem 13, let $S=T-T_{\psi}$; then $\mu_{T}=\mu_{\psi}+$ $\mu_{S}$, where $\mu_{\psi}$ is diffuse (Lemma 4 ) and $\mu_{S}$ is atomic; such a decomposition of a measure is unique [3, Ch. V, $\S 5$, Prop. 15], thus the representation of Theorem 13 is unique: the $\phi_{i}$ are unique and $\psi$ is unique up to an additive constant. One can also write $S$ in the form $S=\sum_{\beta} \phi(\beta) \cdot \beta$, where $\phi: X(\mathscr{R}) \rightarrow C$ and $\sum_{\beta}|\phi(\beta)|<+\infty$.

\section{REFERENCES}

1. N. Bourbaki, Topologie générale, Vols. I, II, Hermann, Paris, 1971, 1974.

2. — Fonctions d'une variable réelle, Chs. I-III, Hermann, Paris, 1949.

3. - Intégration, Chs. I-IV and V, Second edition, Ch. IX, Hermann, Paris, 1965, 1967, 1969.

4. - Théories spectrales, Chs. I-II, Hermann, Paris, 1967.

5. I. Gelfand, D. Raikov and G. Shilov, Commutative Normed Rings, Chelsea, Bronx, N. Y., 1964.

6. L. Gillman and M. Jerison, Rings of Continuous Functions, Springer-Verlag, New York, 1976.

7. E. Hewitt, A problem concerning finitely additive measures, Mat. Tidsskr. B 1951, 81-94 (1951).

8. E. Hewitt and K. Stromberg, Real and Abstract Analysis, Springer-Verlag, New York, 1965.

9. T. H. Hildebrandt, Introduction to the Theory of Integration, Academic, New York, 1963.

10. H. S. Kaltenborn, Linear functional operations on functions having discontinuities of the first kind, Bull. Amer. Math. Soc., 40 (1934), 702-708.

11. H. E. Lacey, The Isometric Theory of Classical Banach Spaces, Springer-Verlag, New York, 1974.

12. A. Pelczynski and Z. Semadeni, Spaces of continuous functions. III, Studia Math., 18 (1959), 211-222.

13. C. E. Rickart, General Theory of Banach Algebras, Krieger, Huntington, N. Y., 1974.

14. H. L. Royden, Real Analysis, Second edition, Macmillan, New York, 1968.

15. W. Rudin, Principles of Mathematical Analysis, Third edition, McGraw-Hill, New York, 1976.

16. — Functional Analysis, McGraw-Hill, New York, 1973.

17. M. H. Stone, Boundedness properties in function-lattices, Canad. J. Math., 1 (1949), 176-186.

18. B. Sz.-Nagy, Introduction to Real Functions and Orthogonal Expansions, Oxford University Press, New York, 1965. 
19. A. Wilansky, Topology for Analysis, Xerox, Lexington, Mass., 1970. Received January 21, 1977.

The University of TeXas at Austin AUstin, TX 78712 


\section{PACIFIC JOURNAL OF MATHEMATICS}

\section{EDITORS}

RICHARD ARENS (Managing Editor)

University of California

Los Angeles, California 90024

C. W. Curtis

University of Oregon

Eugene, OR 97403

C. C. MOORE

University of California

Berkeley, CA 94720

\section{J. DUGUNDJI}

Department of Mathematics University of Southern California Los Angeles, California 90007

R. Finn AND J. Milgram Stanford University Stanford, California 94305

ASSOCIATE EDITORS
E. F. BECKENBACH

B. H. NeUMANN

F. WOLF

K. YoshidA

\section{SUPPORTING INSTITUTIONS}

UNIVERSITY OF BRITISH COLUMBIA UNIVERSITY OF SOUTHERN CALIFORNIA CALIFORNIA INSTITUTE OF TECHNOLOGY STANFORD UNIVERSITY UNIVERSITY OF CALIFORNIA MONTANA STATE UNIVERSITY UNIVERSITY OF TOKYO UNIVERSITY OF NEVADA, RENO UNIVERSITY OF UTAH NEW MEXICO STATE UNIVERSITY OREGON STATE UNIVERSITY UNIVERSITY OF OREGON

WASHINGTON STATE UNIVERSITY UNIVERSITY OF WASHINGTON OSAKA UNIVERSITY 


\section{Pacific Journal of Mathematics}

\section{Vol. 74, No. $1 \quad$ May, 1978}

Gerald Arthur Anderson, Computation of the surgery obstruction groups

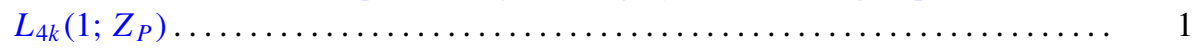

R. K. Beatson, The degree of monotone approximation ................ 5

Sterling K. Berberian, The character space of the algebra of regulated functions . . . 15

Douglas Michael Campbell and Jack Wayne Lamoreaux, Continua in the plane with

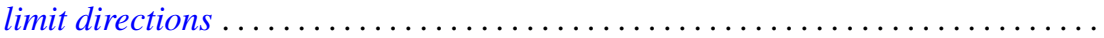

R. J. Duffin, Algorithms for localizing roots of a polynomial and the Pisot

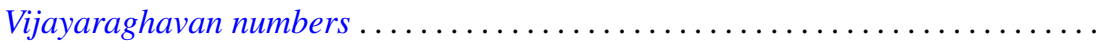

Alessandro Figà-Talamanca and Massimo A. Picardello, Functions that operate on

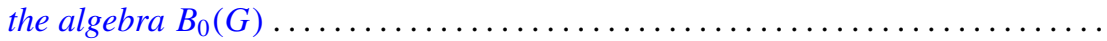

John Erik Fornaess, Biholomorphic mappings between weakly pseudoconvex

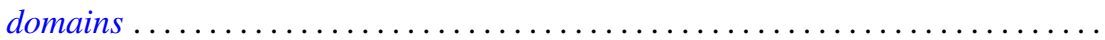

Andrzej Granas, Ronald Bernard Guenther and John Walter Lee, On a theorem of S.

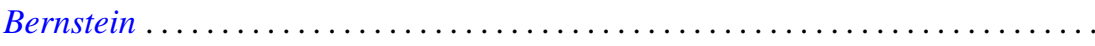

Jerry Grossman, On groups with specified lower central series quotients . .........

William H. Julian, Ray Mines, III and Fred Richman, Algebraic numbers, a constructive development . . . . . . . . . . . . . . . . . . . . . . .

Surjit Singh Khurana, A note on Radon-Nikodým theorem for finitely additive

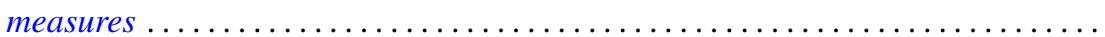

Garo K. Kiremidjian, A Nash-Moser-type implicit function theorem and nonlinear

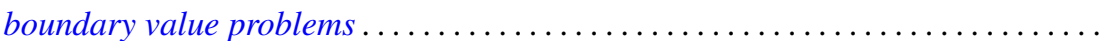

Ronald Jacob Leach, Coefficient estimates for certain multivalent functions ....

John Alan MacBain, Local and global bifurcation from normal eigenvalues. II . . 133

James A. MacDougall and Lowell G. Sweet, Three dimensional homogeneous algebras...

John Rowlay Martin, Fixed point sets of Peano continua ......

R. Daniel Mauldin, The boundedness of the Cantor-Bendixson order of some analytic sets...

Richard C. Metzler, Uniqueness of extensions of positive linear functions ..

Rodney V. Nillsen, Moment sequences obtained from restricted powers . .

Keiji Nishioka, Transcendental constants over the coefficient fields in differential elliptic function fields...

Gabriel Michael Miller Obi, An algebraic closed graph theorem

Richard Cranston Randell, Quotients of complete intersections by $\mathbf{C}^{*}$ actions . . 221

Bruce Reznick, Banach spaces which satisfy linear identities . .

Bennett Setzer, Elliptic curves over complex quadratic fields...

Arne Stray, A scheme for approximating bounded analytic functions on certain subsets of the unit disc.

Nicholas Th. Varopoulos, A remark on functions of bounded mean oscillation and bounded harmonic functions. Addendum to: "BMO functions and the

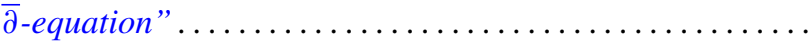

Charles Irvin Vinsonhaler, Torsion free abelian groups quasi-projective over their

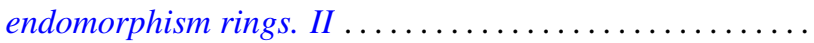

Thomas R. Wolf, Characters of $p^{\prime}$-degree in solvable groups ... 